\title{
Efficacy and adverse events related to the initial dose of methimazole in children and adolescents with Graves' disease
}

\author{
Hyun Gyung Lee, \\ Eun Mi Yang, \\ Chan Jong Kim \\ Department of Pediatrics, Chonnam \\ National University Medical School \\ \& Children's Hospital, Gwangju, \\ Korea
}

Purpose: The first-line antithyroid drug for children and adolescents with Graves' disease (GD) is methimazole (MMI). This study evaluated the relationship between the initial MMI dose and the clinical course of GD after treatment.

Methods: We studied the efficacy of the initial MMI dose and the relationship between the initial MMI dose and adverse events (AEs). We retrospectively enrolled 22 males and 77 females and divided those subjects into 3 groups according to the initial dose of MMl: $<0.4 \mathrm{mg} / \mathrm{kg} /$ day (group $\mathrm{A} ; \mathrm{n}=32$ ); $0.4-0.7 \mathrm{mg} / \mathrm{kg} /$ day (group $B$; $\mathrm{n}=39$ ); and $>0.7 \mathrm{mg} / \mathrm{kg} /$ day (group $\mathrm{C} ; \mathrm{n}=28$ ).

Results: The mean time to the normalization of free thyroxine (fT4) levels upon initial treatment was 5.64, 8.61, and 7.98 weeks in groups $A, B$, and $C$, respectively $(P=0.116)$. The incidence of liver dysfunction, neutropenia, and skin rash was $12.5 \%$, $20.5 \%$, and $42.9 \%$ in groups $A, B$, and $C$, respectively ( $P=0.018)$. Neutropenia, as a severe $A E$, was absent in group $A$, but its prevalence was $7.7 \%$ in group $B$ and $21.4 \%$ in group $C(P=0.015)$. When comparing only groups $B$ and $C$, the incidences of liver dysfunction and neutropenia were higher in group $C(P=0.04$ and $P=0.021$, respectively).

Conclusion: The mean time to the normalization of fT4 levels did not differ among the 3 groups, but the incidence of AEs was higher in the groups that received high $\mathrm{MMI}$ doses. High doses of MMI (>0.7 $\mathrm{mg} / \mathrm{kg} /$ day) should be reconsidered as an initial treatment for children and adolescents with GD.

Keywords: Methimazole, Adverse events, Graves' disease, Child

\section{Highlights}

High doses of MMI did not shorten the time required to normalize fT4, but resulted in the higher incidence of AEs. Low doses of MMI are suitable for mild GD, and high doses of MMI should be reconsidered for initial treatment in children and adolescents with GD.

\section{Introduction}

Graves' disease $(\mathrm{GD})$ is an acquired autoimmune disease that causes diffuse toxic goiters, and it is the most common cause of hyperthyroidism in children and adolescents. ${ }^{1,2}$ ) The 3 treatment options for pediatric GD are the same as those in adults: antithyroid drugs (ATDs), thyroidectomy, and radioactive iodine therapy. ATDs such as propylthiouracil (PTU) methimazole (MMI), and carbimazole (CBZ) are commonly used as initial GD treatments. ${ }^{3}$ All those drugs are thioamide derivatives, and MMI is the active metabolite of CBZ after absorption. ${ }^{4)}$

When ATDs are administered for the initial management of GD in children, PTU should 
be avoided because it carries a risk of severe liver failure. ${ }^{5,6)}$ PTU can cause severe and rapid hepatotoxicity that can require liver transplantation. ${ }^{7)}$ Therefore, MMI is recommended as the initial ATD therapy in children. ${ }^{8,9)}$ The appropriate initial dose of MMI for children has been inadequately investigated. In adults, the initial dose should be adapted to the severity of the hyperthyroidism. Patients with larger goiters and free thyroxine (fT4) levels 3 to 4 times the upper normal limit should be started on $30 \mathrm{mg}$ of MMI daily. Patients with small goiters and lower fT 4 concentrations can be started on 15 to $20 \mathrm{mg}$ of MMI daily. ${ }^{10,11)}$ In children, the typical initial dose used to be 0.5 to 1.0 $\mathrm{mg} / \mathrm{kg} /$ day. ${ }^{3)}$ However, the guidelines of the American Thyroid Association (ATA) and American Association of Clinical Endocrinologists (AACE) say that doses for children can range from 0.1 to $1.0 \mathrm{mg} / \mathrm{kg} / \mathrm{day}$, depending on the initial state of hyperthyroidism. ${ }^{8}$ That initial range is wider than the one typically used in recent years, which tends to be between 0.2 and $0.5 \mathrm{mg} / \mathrm{kg} /$ day. $^{12-14)}$

To date, most publications about medical therapy for GD have focused on the clinical course and adverse events (AEs) in children treated with PTU. Few reports have sought a definite initial dose or examined the clinical course when MMI is used as the initial treatment for children with GD. However, the AEs of MMI, though less dangerous than those of PTU, still require attention and consideration. ${ }^{15,16)}$ Therefore, we retrospectively evaluated the relationship between the initial MMI dose and the development of AEs and investigated the clinical course of GD treated with MMI.

\section{Materials and methods}

\section{Subjects}

This study was conducted at Chonnam National University Hospital (CNUH). We retrospectively reviewed the medical records of children and adolescents who were diagnosed with GD and initially treated with MMI between January 2003 and June 2018 to study the relationship between the initial MMI dose and AEs.

\section{Method}

Based on the ATA guidelines, GD was diagnosed if the following diagnostic criteria were met ${ }^{8}$ : (1) presenting with symptoms of hyperthyroidism, such as tachycardia, excessive sweating, weight loss, or no weight gain; (2) elevated serum triiodothyronine/fT4 levels accompanied by decreased serum thyroid-stimulating hormone (TSH); and (3) positive thyrotropin-binding inhibitory immunoglobulin (TBII). The initial dose was determined by individual physicians based on each patient's clinical symptoms and signs. As the symptoms of hyperthyroidism became more severe, the goiter size reached higher grades in the World Health Organization classification, and the grade of exophthalmos in the ATA classification (based on Werner's NOSPECS classification) increased, higher doses were prescribed. We defined AEs as follows: neutropenia (neutrophil count $<1,000 / \mathrm{mm}^{3}$ ), agranulocytosis (granulocyte count $<250 / \mathrm{mm}^{3}$ ), and liver dysfunction (aspartate aminotransferase $[\mathrm{AST}]$ or alanine aminotransferase [ALT] $>$ $60 \mathrm{IU} / \mathrm{L}) .{ }^{17)}$ After the start of MMI treatment, AEs were assessed at least once every 2-4 weeks until the serum fT4 levels fell to within the normal range. During maintenance treatment, after the normalization of serum fT4 levels, laboratory evaluations were conducted more than 3 times a year. MMI treatment was withdrawn if thyroid function was maintained within the normal range during 18-24 months of maintenance treatment or if the level of TBII descended as a negative finding. ${ }^{14)}$ After the completion of MMI treatment, a thyroid function test was performed more than twice a year for at least 2 years to check for relapses. The outcomes were evaluated more than 2 years after the initiation of MMI treatment. We enrolled 99 patients in this study and divided the subjects into 3 groups according to their initial dose of MMI: $<0.4 \mathrm{mg} / \mathrm{kg} /$ day (group A; $\mathrm{n}=32$ ); $0.4-0.7 \mathrm{mg} / \mathrm{kg} /$ day (group B; $\mathrm{n}=39$ ); and $>0.7 \mathrm{mg} / \mathrm{kg} /$ day (group $\mathrm{C} ; \mathrm{n}=28$ ). We analyzed baseline characteristics, such as age, sex, family history of thyroid disease, and AEs, through a retrospective analysis of medical records. In addition, we evaluated serum levels of fT4, TSH, complete blood count, AST, ALT, anti-thyroid peroxidase antibody (anti-TPO), antithyroglobulin antibody (ATA), and TBII. FT4 (normal range, $0.89-1.76 \mathrm{ng} / \mathrm{dL}$; measurable range up to $12.0 \mathrm{ng} / \mathrm{dL}$ ) and TSH (normal range, $0.64-6.27 \mathrm{mIU} / \mathrm{L}$ ) were measured using a chemiluminescence immunoassay technique (ADVIA Centaur, Siemens, Germany). Anti-TPO (normal range, 0-34 IU/mL) and ATA (normal range, $0-115 \mathrm{IU} / \mathrm{mL}$ ) were analyzed using an electrochemiluminescence immunoassay kit (COBAS, Roche Diagnostics GmbH, Mannheim, Germany). TBII values were presented as percentages from 2003 to 2013 (normal range, 0\%-15\%) using a radioimmunoassay (RSR Ltd., Cardiff, UK) and then presented as IU/L (normal range, 0-1.5 IU/L) using a different radioimmunoassay (Shinjin Medic Inc., Goyang, Korea), so it is segregated into 2 categories.

\section{Statistical analysis}

Data are expressed as the mean \pm standard deviation or proportion (\%) depending on the characteristics of the variables. A 1-way analysis of variance was used to compare the means of continuous variables (age, sex ratio, MMI dose, fT4, TSH, TBII, anti-TPO, ATA, time required for normalization of fT4), and post hoc Tukey tests were used to compare group differences. We used the Pearson chi-square test to compare the proportions of categorical variables (incidence of AEs) among different patient subgroups. All statistical analyses were performed using IBM SPSS Statistics ver. 21.0 (IBM Co., Armonk, NY, USA). P-values $<0.05$ were considered statistically significant. 


\section{Results}

\section{Comparison of MMl efficacy based on the initial dose}

We enrolled 99 patients (22 males and 77 females) and divided them into 3 groups: A $(n=32), B(n=39)$, and C $(n=28)$. The mean age, sex ratio, mean MMI dose, serum fT4 and TSH concentrations, TBII, anti-TPO, and ATA at diagnosis are shown in Table 1. The fT4 level at diagnosis was $2.40 \mathrm{ng} / \mathrm{dL}$ in group A, $4.30 \mathrm{ng} / \mathrm{dL}$ in group B, and $4.69 \mathrm{ng} / \mathrm{dL}$ in group C. The fT4 level was significantly higher in group $\mathrm{C}$, indicating that patients who presented with greater disease severity were started on higher MMI doses $(P=0.001)$. However, when comparing only groups $\mathrm{B}$ and $\mathrm{C}$, the $\mathrm{fT} 4$ and TSH levels did not differ significantly (fT4, $P=0.710$; TSH, $P=1.000$ ). The levels of TBII, anti-TPO, and ATA did not differ significantly among the groups.

The time required to normalize serum $\mathrm{fT} 4$ levels after the initial treatment was used to assess the efficacy of MMI. The mean duration was $5.6 \pm 4.2,8.6 \pm 10.4$, and $7.9 \pm 8.2$ weeks in groups $A, B$, and $C$, respectively (Fig. 1). No significant differences were found among the 3 groups $(P=0.116)$, and no statistical differences were found when comparing only groups $\mathrm{B}$ and $\mathrm{C}$, either. Therefore, higher doses did not shorten the time required to normalize fT4.

\section{Comparison of the $A E$ incidence and clinical course}

During the observation period, no serious AEs, such as agranulocytosis or severe liver failure, were observed. The proportion of patients with AEs was higher in group $\mathrm{C}$ than in the other groups $(12.5 \%, 20.5 \%$, and $42.9 \%$ in groups $\mathrm{A}, \mathrm{B}$, and $\mathrm{C}$, respectively, $P=0.018$ ) (Table 2 ). The incidence of liver dysfunction was significantly higher in group $\mathrm{C}$ than in the other groups $(9.4 \%, 7.7 \%$, and $28.6 \%$ in groups $\mathrm{A}, \mathrm{B}$, and $\mathrm{C}$, respectively, $P=0.034$ ). Neutropenia was not observed in group $\mathrm{A}$, but it occurred in $7.7 \%$ of the patients in group $\mathrm{B}$ and $21.4 \%$ of those in group $\mathrm{C}(P=0.015)$. The incidence of skin rash was also higher in the groups with higher MMI doses $(3.1 \%$, $5.1 \%$, and $10.7 \%$ in groups A, B, and C, respectively), but those differences were not significant (Table 2). When comparing only groups $\mathrm{B}$ and $\mathrm{C}$, the incidence of liver dysfunction and neutropenia was significantly higher in group $C(P=0.04$ and $P=0.021$, respectively).

Patients with clinical conditions such as viral infections and those requiring other medical treatments that could be confused with the development of AEs due to MMI were excluded from this statistical analysis. If mild AEs, such as a skin rash

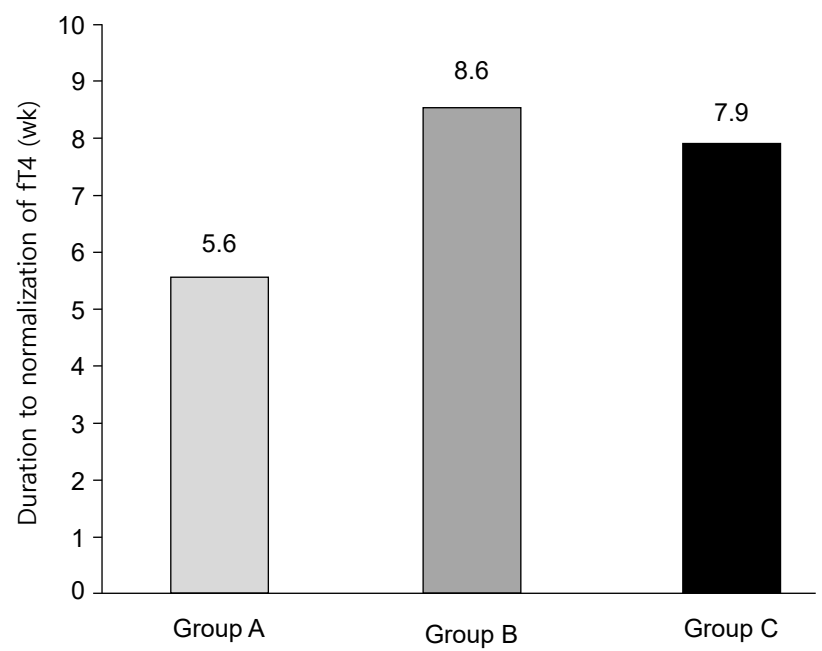

Fig. 1. The time required to normalize free T4 levels after initiating methimazole treatment in patients with Graves' disease. The mean duration was 5.6 \pm 4.2 , 8.6 \pm 10.4 , and $7.9 \pm 8.2$ weeks in groups $A, B$, and $C$, respectively $(P=0.116)$. Group A, initial dose of $M M l<0.4 \mathrm{mg} / \mathrm{kg} /$ day; group $\mathrm{B}, 0.4-0.7 \mathrm{mg} / \mathrm{kg} /$ day; group $C_{\text {, }}>0.7$ $\mathrm{mg} / \mathrm{kg} / \mathrm{day}$.

Table 1. Clinical characteristics and laboratory findings at diagnosis

\begin{tabular}{|c|c|c|c|c|}
\hline Characteristic & Group A $(n=32)$ & Group B (n=39) & Group C $(n=28)$ & $P$-value \\
\hline Age (yr) & $12.2 \pm 3.6$ & $12.7 \pm 2.6$ & $11.2 \pm 3.2$ & 0.128 \\
\hline Male sex & $5(15.6)$ & $11(28.2)$ & $6(21.4)$ & 0.452 \\
\hline MMI dose (mg/kg/day) & $0.22 \pm 0.09^{\dagger, \S}$ & $0.54 \pm 0.10^{\ddagger}$ & $1.02 \pm 0.27$ & $0.000^{*}$ \\
\hline MMI daily total dose (mg/day) & $9.65 \pm 5.56^{\dagger, \S}$ & $24.74 \pm 7.78^{\ddagger}$ & $37.86 \pm 14.16$ & $0.000^{*}$ \\
\hline Free T4 (ng/dL) & $2.40 \pm 1.04^{\dagger, \S}$ & $4.30 \pm 2.06$ & $4.69 \pm 2.62$ & $0.001^{*}$ \\
\hline TSH (mIU/L) & $1.05 \pm 2.93^{t, \S}$ & $0.013 \pm 0.02$ & $0.008 \pm 0.004$ & $0.018^{*}$ \\
\hline \multicolumn{5}{|l|}{ TBII } \\
\hline IU/L (normal range, 0-1.5) & $12.47 \pm 36.13$ & $22.68 \pm 38.73$ & $13.84 \pm 21.46$ & 0.610 \\
\hline$\%$ (normal range, $0-15$ ) & $29.50 \pm 30.65$ & $39.15 \pm 30.53$ & $45.90 \pm 29.72$ & 0.367 \\
\hline Anti-TPO (IU/mL) & $22(68.7)$ & $25(64.1)$ & $14(50.0)$ & 0.248 \\
\hline ATA $(I U / m L)$ & $26(81.3)$ & $33(84.6)$ & $23(82.1)$ & 0.278 \\
\hline
\end{tabular}

Values are presented as mean \pm standard deviation and number (\%).

Group A, initial dose of MMl<0.4 mg/kg/day; group B, 0.4-0.7 mg/kg/day; group C, >0.7 mg/kg/day; MMI, methimazole; TSH, thyroid-stimulating hormone; TBII, thyrotropin-binding inhibitory immunoglobulin; Anti-TPO, antithyroid peroxidase antibody; ATA, antithyroglobulin antibody.

${ }^{*} P<0.05$, statistical significances were tested by 1 -way analysis of variance. Post hoc test: ${ }^{\dagger} P<0.05$, group A vs. group $B ;{ }^{\ddagger} P<0.05$, group $B$ vs. group $C ;{ }^{\S} P<0.05$, group A vs. group $C$. 
resolved by antihistamines or liver dysfunction, occurred, the clinical course was observed without reducing the MMI dose. If the AEs continued or proved burdensome for the patient, the MMI dose was reduced. The onset of severe hepatitis (AST or ALT elevation more than 3 times the upper normal limit) or agranulocytosis requires the termination of MMI treatment. Overall, when AEs appeared, the initial MMI dose was maintained in $66.7 \%$ of cases and reduced in $33.3 \%$ of cases. The rate of dose reduction due to AEs was $50.0 \%$ in group B and $33.3 \%$ in group C. No patients in group A received an MMI dose reduction. When neutropenia occurred, the dose of MMI was reduced, and neutrophil counts were monitored closely.

The time from the start of MMI therapy to the appearance of AEs was $1.68,2.03$, and 0.75 months for liver dysfunction, neutropenia, and skin rash, respectively. Those times did not differ significantly among the groups. The clinical courses of patients $>2$ years after the initiation of MMI are shown in Table 3. The patients whose observation period was less than 2 years and those who were transferred to another hospital or lost to follow-up were excluded from this statistical analysis. The average observation period was 40.5 months. The overall remission rate was $34.7 \%$, and the remission rates did not differ significantly among the groups $(37.5 \%, 31.0 \%$, and $36.8 \%$ in groups A, B, and C, respectively). The overall percentage of patients who failed to withdraw from MMI therapy was 30.6\% $(29.1 \%, 37.9 \%$, and $21.1 \%$ in groups A, B, and C, respectively). The overall rate of relapse after MMI treatment was withdrawn was $31.9 \%(29.1 \%, 27.6 \%$, and $42.1 \%$ in groups A, B, and $\mathrm{C}$, respectively). The average period to relapse after MMI withdrawal was 4.6 months.

\section{Discussion}

ATDs have been used to treat GD for 6 decades. ${ }^{4)}$ MMI is prioritized over PTU as an initial treatment for GD for 2 reasons. The first is that compliance with MMI treatment is high among children and adolescents because of its once-a-day dosage. ${ }^{8)}$ MMI has a longer half-life than PTU and is effective when given as a single daily dose. ${ }^{18)}$ The second reason is the reduced risk of major AEs, compared with those of PTU. PTU can cause fulminant hepatic necrosis, which can be fatal, and liver transplantation has been necessary in a few patients taking PTU. ${ }^{19)}$ Although liver dysfunction has also appeared in patients taking MMI, as in our study, the hepatotoxicity associated with MMI treatment is typically cholestatic, and hepatocellular disease is rarely seen. ${ }^{20)}$ Therefore, MMI should be used as the first-line drug in patients diagnosed with GD. However, few studies have assessed the relationship between the initial MMI dose and AEs.

ATD treatment can use the titration or block and replace method. ${ }^{21,22)}$ The block and replace method is not commonly used because it requires higher doses and has thus been shown to result in a higher rate of side effects. ${ }^{8)}$ The ATA and AACE guidelines recommend the titration method. ${ }^{8)}$ However, those guidelines offer wide ranges, which differ slightly from each other, for the initial ATD dose: 10-20 mg daily for adults and $0.1-1.0 \mathrm{mg} / \mathrm{kg} /$ day in children. In Japan, Minamitani et al. ${ }^{14)}$ recommended starting doses of $0.25-1.0 \mathrm{mg} / \mathrm{kg} /$ day in children, which is lower than the dose generally used in Japan (0.5-1.0 $\mathrm{mg} / \mathrm{kg} /$ day). ${ }^{23)}$ There is no definitive guideline for the initial MMI dose for childhood-onset GD in Korea. Since the recent designation of AE reduction as the highest priority, ATD doses have tended to decrease. ${ }^{14)}$ In our study, based on 15 years of data, we divided the range from 0.1 to $1.0 \mathrm{mg} / \mathrm{kg} /$ day into 3

Table 2. Adverse events associated with methimazole (MMI) therapy

\begin{tabular}{lccccc}
\hline Adverse event & Group A $(n=32)$ & Group B $(n=39)$ & Group C $(n=28)$ & Total patients & $P$-value \\
\hline Liver dysfunction & $3(9.4)$ & $3(7.7)$ & $8(28.6)$ & $14(14.1)$ & $0.034^{*}$ \\
Neutropenia & $0(0)$ & $3(7.7)$ & $6(21.4)$ & $9(9.1)$ & $0.015^{*}$ \\
Skin rash & $1(3.1)$ & $2(5.1)$ & $3(10.7)$ & $6(6.1)$ & 0.447 \\
Total adverse events & $4(12.5)$ & $8(20.5)$ & $12(42.9)$ & $24(24.2)$ & $0.018^{*}$ \\
\hline
\end{tabular}

Values are presented as number (\%).

Group A, initial dose of MMl<0.4 mg/kg/day; group B, 0.4-0.7mg/kg/day; group C, $>0.7 \mathrm{mg} / \mathrm{kg} /$ day.

${ }^{*} P<0.05$, statistical significances using a chi-square test among 3 groups.

Table 3. Outcomes in patients with Graves' disease followed more than 2 years after the initiation of methimazole (MMI)

\begin{tabular}{lcccc}
\hline Variable & Group A $(n=24)$ & Group B $(n=29)$ & Group C $(n=19)$ & Total $(n=72)$ \\
\hline Observation period $(\mathrm{mo})$ & $40.3 \pm 23.6$ & $32.5 \pm 20.4$ & $52.8 \pm 24.5$ & $40.5 \pm 23.7$ \\
Remission & $9(37.5)$ & $9(31.0)$ & $7(36.8)$ & $25(34.7)$ \\
No remission & $15(62.5)$ & $20(68.9)$ & $12(63.2)$ & $47(65.3)$ \\
$\quad$ No withdrawal of MMI & $7(29.1)$ & $11(37.9)$ & $4(21.1)$ & $22(30.6)$ \\
$\quad$ Relapse after withdrawal of MMI & $7(29.1)$ & $8(27.6)$ & $8(42.1)$ & $23(31.9)$ \\
Duration of MMI withdrawal before relapse (mo) & $4.1 \pm 3.9$ & $5.4 \pm 2.7$ & $4.1 \pm 1.2$ & $4.6 \pm 2.8$ \\
Thyroidectomy & $1(4.1)$ & $1(3.4)$ & $0(0)$ & $2(2.8)$ \\
\hline
\end{tabular}

Values are presented as mean \pm standard deviation and number (\%).

Group A, initial dose of MMl<0.4 mg/kg/day; group B, 0.4-0.7mg/kg/day; group C, $>0.7 \mathrm{mg} / \mathrm{kg} /$ day. 
sections to study the relationship between the initial MMI dose and the clinical course after treatment.

The initial MMI dose is determined by the severity of GD, but few studies to date have focused on children. The severity of GD is assessed using fT4 and TSH levels, with higher fT4 levels and lower TSH levels indicating higher GD severity. ${ }^{24)}$ In adults, Nakamura et al. ${ }^{11)}$ reported that $15 \mathrm{mg} /$ day of MMI was appropriate for mild and moderate GD (fT4 level less than $7 \mathrm{ng} / \mathrm{dL}$ ) because it offered similar effectiveness and a lower frequency of adverse reactions compared with $30 \mathrm{mg} /$ day, whereas $30 \mathrm{mg} /$ day of MMI was appropriate for severe GD (fT4 level more than $7 \mathrm{ng} / \mathrm{dL}$ ). Shiroozu et al. ${ }^{25)}$ also reported that greater efficacy from higher MMI doses was clear in severe GD, but in mild GD, $30 \mathrm{mg} /$ day of MMI was no more effective than $15 \mathrm{mg} /$ day. In children, previous studies have produced contrasting results. Slyper et al. ${ }^{26)}$ reported that an initial MMI dose $>0.5 \mathrm{mg} / \mathrm{kg}$ was more effective for children and adolescents with GD than doses $<0.5 \mathrm{mg} / \mathrm{kg}$, without considering GD severity. Matsushita et al. $^{23)}$ reported that the mean time to the normalization of fT4 levels did not differ significantly between groups using a high $(\geq 0.7 \mathrm{mg} / \mathrm{kg} /$ day $)$ and low $(<0.7 \mathrm{mg} / \mathrm{kg} /$ day $)$ dose of MMI, and their groups did not differ significantly in GD severity.

In our study, the severity of GD was significantly higher in the moderate-dose (group B) and high-dose (group C) groups than in the low-dose group (group A). In the low-dose group, because the severity of GD was low, the time to euthyroid was shorter than that in the other groups, despite using a lower dose. Therefore, for mild GD, a low dose $(<0.4 \mathrm{mg} / \mathrm{kg} /$ day $)$ of MMI is appropriate. Although the difference was not statistically significant, the remission rate in the low-dose group was also slightly higher because that group included many cases of mild GD. When we compared the 3 groups in our study, they did not differ significantly in the time required to reach a euthyroid state after MMI initiation. In addition, long-term outcomes, such as the remission and relapse rates, did not differ significantly among the groups.

Several reports have indicated that MMI-induced AEs depend on the MMI dose. ${ }^{18)}$ In adults, the frequency of AEs can be dose-related, and the rate of severe AEs is very low in patients receiving MMI doses $<10 \mathrm{mg}$ /day. ${ }^{4)}$ In another study in adults, MMI-induced neutropenia was significantly higher in patients receiving $30 \mathrm{mg} /$ day than in those receiving $15 \mathrm{mg} /$ day. ${ }^{27)}$ These results are similar to the outcomes of our study in children. In our data, the frequency of MMI-induced AEs was significantly higher in the high-dose group (42.9\%) than in the low- (12.5\%) and moderate-dose $(20.5 \%)$ groups, suggesting that the AEs were dose-dependent. A higher dose of MMI resulted in a higher frequency of major AEs, such as liver dysfunction and neutropenia. Neutropenia occurred in $7.7 \%$ of the moderatedose group and $21.4 \%$ in the high-dose group, and it did not occur at all in the low-dose group. Sato et al. ${ }^{17)}$ reported that when 64 MMI-treated children were divided into 3 groups by dose, the frequency of AEs in the high-dose group was extremely high (63\%), consistent with our results.
When we compared only the moderate- and high-dose groups, we found no difference in the remission rate between the 2 groups. But the incidence of AEs was significantly higher in the high-dose group. Therefore, the use of high MMI doses $(>0.7 \mathrm{mg} / \mathrm{kg} / \mathrm{day})$ as an initial therapy should be avoided in children and adolescents with GD. We find that low MMI doses are suitable for children with mild GD, and moderate doses of MMI are appropriate for children with severe GD. In addition, to ensure patient understanding, we recommend that patients be informed both verbally and in writing about MMI-associated AEs.

In our study, the time from the start of MMI therapy to the appearance of AEs was approximately 1.5 months. Skin rash appeared in 0.8 months, which tended to be sooner than other AEs, and neutropenia appeared in 2.0 months, which was longer than it took other AEs to develop. AEs developed within 3 months of treatment initiation in $79.2 \%$ of AE cases. Ohye et $\mathrm{al}^{28)}$ reported that most AEs (91.6\%) occurred within the first 3 months of ATD treatment. Therefore, routine monitoring and short-term follow-up should be performed during that period. Agranulocytosis, which is a serious AE, was not observed in our study, but neutropenia was observed in $9.1 \%$ of cases. Yasuda et al. ${ }^{29)}$ reported that neutropenia was the most common AE to occur after 1 year, and it tended to appear later than other AEs. Therefore, monitoring for the development of neutropenia should be continued throughout MMI therapy.

We recognize that the limitations of our study are its retrospective nature, the small number of patients, and the nonrandom allocation of patients to the MMI dose-based groups. A long-term, double-blind, prospective trial with wellmatched patient groups should be conducted to confirm our findings.

In conclusion, we reviewed the medical records of children and adolescents with GD to investigate the efficacy of MMI according to the initial dose and examine the relationship between the initial MMI dose and the development of AEs. Low MMI doses are suitable for treating mild GD, and moderate doses of MMI are advisable for severe GD. High MMI doses ( $>0.7 \mathrm{mg} / \mathrm{kg} /$ day) should be reconsidered as an initial treatment in children and adolescents with GD. Most AEs were observed within 3 months of MMI treatment initiation, so close monitoring of AEs is required during that period.

\section{Ethical statement}

This study was approved by the Institutional Review Board of Chonnam National University Hospital (CNUH-2020-340).

\section{Conflict of interest}

No potential conflict of interest relevant to this article was reported. 


\section{References}

1. Metso S, Jaatinen P, Salmi J. Graves' disease. N Engl J Med 2008;359:1408-9.

2. Weetman AP. Graves' disease. N Engl J Med 2000;343:123648 .

3. Rivkees SA. The treatment of Graves' disease in children. J Pediatr Endocrinol Metab 2006;19:1095-112.

4. Cooper DS. Antithyroid drugs. N Engl J Med 2005;352:90517.

5. Rivkees SA, Mattison DR. Propylthiouracil hepatoxicity in children and recommendations for discontinuation of use. Int J Pediatr Endocrinol 2009;2009:132041.

6. Rivkees SA, Mattison DR. Ending propylthiouracil-induced liver failure in children. N Engl J Med 2009;360:1574-5.

7. Karras S, Memi E, Kintiraki E, Krassas GE. Pathogenesis of propylthiouracil-related hepatotoxicity in children: present concepts. J Pediatr Endocrinol Metab 2012;25:623-30.

8. Bahn RS, Burch HB, Cooper DS, Garber JR, Greenlee $\mathrm{MC}$, Klein I, et al. Hyperthyroidism and other causes of thyrotoxicosis: management guidelines of the American Thyroid Association and American Association of Clinical Endocrinologists. Thyroid 2011;21:593-646.

9. He CT, Hsieh AT, Pei D, Hung YJ, Wu LY, Yang TC, et al. Comparison of single daily dose of methimazole and propylthiouracil in the treatment of Graves' hyperthyroidism. Clin Endocrinol (Oxf) 2004;60:676-81.

10. Corvilain B, Hamy A, Brunaud L, Borson-Chazot F, Orgiazzi J, Bensalem H, et al. Treatment of adult Graves' disease. Ann Endocrinol 2018;79:618-35.

11. Nakamura H, Noh JY, Itoh K, Fukata S, Miyauchi A, Hamada N, et al. Comparison of methimazole and propylthiouracil in patients with hyperthyroidism caused by Graves' disease. J Clin Endocrinol Metab 2007;92:2157-62.

12. Lee HS, Hwang JS. The treatment of Graves' disease in children and adolescents. Ann Pediatr Endocrinol Metab 2014;19:122-6.

13. Dötsch J, Rascher W, Dörr HG. Graves' disease in childhood: a review of the options for diagnosis and treatment. Paediatr Drugs 2003;5:95-102.

14. Minamitani K, Sato H, Ohye H, Harada S, Arisaka O. Guidelines for the treatment of childhood-onset Graves' disease in Japan, 2016. Clin Pediatr Endocrinol 2017;26:2962.

15. Yu W, Wu N, Li L, Wang J, OuYang H, Shen H. Side effects of propylthiouracil and methimazole in the treatment of hyperthyroidism: a systematic review and meta-analysis. Endocr Pract 2020;26:207-17.

16. Kim HK, Yoon JH, Jeon MJ, Kim TY, Shong YK, Lee MJ, et al. Characteristics of Korean patients with antithyroid drug- induced agranulocytosis: a multicenter study in Korea. Endocrinol Metab 2015;30:475-80.

17. Sato H, Sasaki N, Minamitani K, Minagawa M, Kazukawa I, Sugihara S, et al. Higher dose of methimazole causes frequent adverse effects in the management of Graves' disease in children and adolescents. J Pediatr Endocrinol Metab 2012;25:863-7.

18. Kaguelidou F, Carel JC, Léger J. Graves' disease in childhood: advances in management with antithyroid drug therapy. Horm Res Paediatr 2009;71:310-7.

19. Ruiz JK, Rossi GV, Vallejos HA, Brenet RW, Lopez IB, Escribano AA. Fulminant hepatic failure associated with propylthiouracil. Ann Pharmacother 2003;37:224-8.

20. Woeber KA. Methimazole-induced hepatotoxicity. Endocr Pract 2002;8:222-4.

21. Muldoon BT, Mai VQ, Burch HB. Management of Graves' disease: an overview and comparison of clinical practice guidelines with actual practice trends. Endocrinol Metab Clin North Am 2014;43:495-516.

22. Abraham P, Avenell A, McGeoch S, Clark L, Bevan J. Antithyroid drug regimen for treating Graves hyperthyroidism. Cochrane Database Syst Rev 2010;2010:CD003420.

23. Matsushita R, Nakagawa Y, Nagata E, Satake E, Sano S, Yamaguchi R, et al. Initial treatment of pediatric Graves' disease with methimazole: a retrospective follow-up study. Clin Pediatr Endocrinol 2010;19:101-8.

24. Cooper DS. Hyperthyroidism. Lancet 2003;362:459-68.

25. Shiroozu A, Okamura K, Ikenoue H, Sato K, Nakashima T, Yoshinari M, et al. Treatment of hyperthyroidism with a small single daily dose of methimazole. J Clin Endocrinol Metab 1986;63:125-8.

26. Slyper AH, Wyatt D, Boudreau C. Effective methimazole dose for childhood Graves' disease and use of free triiodothyronine combined with concurrent thyroidstimulating hormone level to identify mild hyperthyroidism and delayed pituitary recovery. J Pediatr Endocrinol Metab 2005; 18:597-602.

27. Takata K, Kubota S, Fukata S, Kudo T, Nishihara E, Ito M, et al. Methimazole-induced agranulocytosis in patients with Graves' disease is more frequent with an initial dose of 30 mg daily than with $15 \mathrm{mg}$ daily. Thyroid 2009;19:559-63.

28. Ohye H, Minagawa A, Noh JY, Mukasa K, Kunii Y, Watanabe N, et al. Antithyroid drug treatment for Graves' disease in children: a long-term retrospective study at a single institution. Thyroid 2014;24:200-7.

29. Yasuda K, Miyoshi Y, Tachibana M, Namba N, Miki K, Nakata Y, et al. Relationship between dose of antithyroid drugs and adverse events in pediatric patients with Graves' disease. Clin Pediatr Endocrinol 2017;26:1-7. 